\title{
INCLUSION THEOREMS FOR SONNENSCHEIN MATRICES ${ }^{1}$
}

\author{
FREDERICK HARTMANN
}

1. Introduction. Inclusion theorems for various methods of summability have been the subject of recent research [2], [5]. In this article necessary and sufficient conditions for the inclusion of Sonnenschein matrix methods are investigated with special attention to matrices with complex entries.

Let $f$ be a function that is analytic for $z \in D_{f}=\{z:|:|<R\}, R>1$ and $f(1)=1$. Let

$$
\begin{array}{rlrl}
\{f(z)\}^{n} & =\sum_{k=0}^{\infty} a_{n k} z^{k} & n & =1,2, \cdots, \\
a_{00} & =1, \quad a_{0 k}=0 & k & =1,2, \cdots .
\end{array}
$$

Then $f$ determines a sequence to sequence transformation, $A(f)$ $=\left(a_{n k}\right)$, whereby if $\left\{s_{k}\right\}$ is a sequence and $\sigma_{n}=\sum_{k=0}^{\infty} a_{n k} s_{k}, n=0,1$, $2, \cdots$ with $\sigma_{n} \rightarrow \sigma$ then $\left\{s_{k}\right\}$ is said to be $A(f)$-summable to $\sigma$. Such matrices are called Sonnenschein matrices [7]. Special well-known cases to be discussed here are the Taylor or Circle method, $T(r)$ [8], $f(z)=(1-r) z /(1-r z),|r|<1$; the Laurent method, $S(q)$ [8], $f(z)$ $=(1-q) /(1-q z),|q|<1$; the Euler-Knopp method, $E(p)$ [1], $f(z)$ $=(1-p)+p z ;$ and a generalization of the three preceding, the Karamata method, $K(\alpha, \beta)[7], f(z)=\{\alpha+(1-\alpha-\beta) z\} /(1-\beta z)$, $|\beta|<1$. In this new notation $T(r)=K(0, r), S(q)=K(1-q, q), E(p)$ $=K(1-p, 0)$. Necessary and sufficient conditions for these methods to be regular are $0 \leqq r<1,[3] ; 0<q<1,[4] ; 0<p \leqq 1,[1]$; and $\alpha=\beta=0$ or $1-|\alpha|^{2}>(1-\bar{\alpha})(1-\beta)>0$, [6] respectively.

The following lemma and notation will be used in the sequel.

Leмma 1. Let $D A(f)$ denote the domain of values $z$ for which the geometric series is $A(f)$-summable to $(1-z)^{-1}$. Then

$$
D A(f)=\{z:|f(z)|<1\}, \quad z \in D_{f} .
$$

Proof. Let the $n$th partial sum of the geometric series be denoted by $S_{n}=\left(1-z^{n+1}\right) /(1-z)$. Then the $A(f)$-transform, $\left\{\sigma_{n}\right\}$, of $\left\{S_{n}\right\}$ is given by

Presented to the Society, January 24,1969; received by the editors August 7, 1968.

${ }^{1}$ This work was part of the author's doctoral dissertation completed at Lehigh University, 1968, under the direction of Professor J. P. King. 


$$
\begin{aligned}
\sigma_{n} & =\sum_{k=0}^{\infty} a_{n k} S_{k}=(1-z)^{-1} \sum_{k=0}^{\infty} a_{n k}-(1-z)^{-1} \sum_{k=0}^{\infty} a_{n k} z^{k+1} \\
& =(1-z)^{-1}-z(1-z)^{-1}\{f(z)\}^{n} .
\end{aligned}
$$

Thus $\sigma_{n} \rightarrow(1-z)^{-1}$ if and only if $[f(z)]^{n} \rightarrow 0$ if and only if $|f(z)|<1$. Let

$$
\begin{aligned}
m & =\left\{x=\left\{x_{n}\right\}: x \text { is bounded }\right\}, \quad c=\left\{x=\left\{x_{n}\right\}: x \text { is convergent }\right\}, \\
c_{A(f)} & =\left\{x: A(f) x=\left\{\sum_{k=0}^{\infty} a_{n k} x_{k}\right\} \in c\right\}, \quad \bar{\Delta}(0,1)=\{z:|z| \leqq 1\} .
\end{aligned}
$$

\section{Products and inverses.}

Theorem 1. Suppose $A(f), A(g)$ are Sonnenschein matrices and $\mathrm{g}(\bar{\Delta}(0,1)) \subset D_{f}$ then $A(f) \cdot A(g)=A(f \circ g)$ and moreover $(A(f) A(g)) y$ $=A(f)(A(g) y)$ for all $y \in m$.

Proof. Let $z \in \bar{\Delta}(0,1)$ and $A(f)=\left(a_{n k}\right), A(g)=\left(b_{k j}\right)$. Then

$$
\{g(z)\}^{k}=\sum_{j=0}^{\infty} b_{k j} z^{j}, \quad k=0,1,2, \cdots
$$

and the convergence is absolute. Since $g(z) \in D_{f}$

$$
\begin{aligned}
\{f(g(z))\}^{n} & =\sum_{k=0}^{\infty} a_{n k}\{g(z)\}^{k}, \quad n=0,1,2, \cdots \\
& =\sum_{k=0}^{\infty} a_{n k}\left(\sum_{j=0}^{\infty} b_{k j} z^{j}\right)=\sum_{j=0}^{\infty}\left(\sum_{k=0}^{\infty} a_{n k} b_{k j}\right) z^{j} .
\end{aligned}
$$

The rearrangement (1) is permitted since the series involved converge absolutely. Likewise $f(g(z))$ is analytic on $D_{f \circ o} \supset \bar{\Delta}(0,1)$ and hence

$$
\{f(g(z))\}^{n}=\sum_{k=0}^{\infty} c_{n k} z^{k}, \quad n=0,1,2, \cdots
$$

Thus by (1) and the uniqueness of power series representation

$$
c_{n k}=\sum_{j=0}^{\infty} a_{n j} b_{j k}, \quad n=0,1,2, \cdots, \quad k=0,1,2, \cdots
$$

If $y \in m$, there exists $M$, such that $\left|y_{j}\right|<M$, for all $j$ and

since

$$
\sum_{k=0}^{\infty} a_{n k}\left(\sum_{j=0}^{\infty} b_{k j} y_{j}\right)=\sum_{j=0}^{\infty}\left(\sum_{k=0}^{\infty} a_{n k} b_{k j}\right) y_{j}
$$




$$
\sum_{k=0}^{\infty}\left|a_{n k}\right|\left(\sum_{j=0}^{\infty}\left|b_{k j}\right|\left|y_{j}\right|\right) \leqq M \sum_{k=0}^{\infty}\left|a_{n k}\right| \sum_{j=0}^{\infty}\left|b_{n j}\right|
$$

and the right-hand side converges since $1 \in D_{\text {fog }}$

In the following corollary $f$ is a one-to-one analytic function and $D_{f^{-1}}$ is the disk about the origin on which $f^{-1}$ has a power series representation. (These two conditions are summarized in the single hypothesis $A(f)$ and $A\left(f^{-1}\right)$ are Sonnenschein matrices.)

Corollary. Suppose $A(f)$ and $A\left(f^{-1}\right)$ are Sonnenschein matrices with $D_{f^{-1}} \supset f(\bar{\Delta}(0,1))$ then $A(f) \cdot A\left(f^{-1}\right)=I=A\left(f^{-1}\right) \cdot A(f)$, where $I$ is the identity matrix, i.e. $A\left(f^{-1}\right)=\{A(f)\}^{-1}$.

Proof. $A(f)$ and $A\left(f^{-1}\right)$ Sonnenschein imply $D_{f} \supset \bar{\Delta}(0,1)$ and $D_{f^{-1}} \supset \bar{\Delta}(0,1)$. Furthermore, $D_{f} \supset f^{-1}(\bar{\Delta}(0,1))$ and $D_{f^{-1}} \supset f(\bar{\Delta}(0,1))$. Therefore $A(f) \cdot A\left(f^{-1}\right)=A\left(f \circ f^{-1}\right)=A(e)=I=A(e)=A\left(f^{-1} \circ f\right)$ $=A\left(f^{-1}\right) \cdot A(f)$, where $e$ is the identity function on the domain of $f \circ f^{-1}$ and $f^{-1} \circ f$ respectively.

3. Inclusion theorems. The following theorem can easily be proved using infinite matrix algebra.

Theorem 2. Let $A, B$ be one-to-one sequence matrix transformations. Let $A^{-1}$ exist and $B\left(A^{-1} y\right)=\left(B A^{-1}\right) y$ and $A\left(A^{-1} y\right)=\left(A A^{-1}\right) y=y$, for all $y \in m$. Then $c_{A} \subset c_{B}$ if and only if $B A^{-1}$ is conservative. Moreover, if $A$ is regular then $A x$ and $B x$ converge to the same limit for $x \in c_{A}$ if and only if $B A^{-1}$ is regular.

We are now prepared to prove our main result.

Theorem 3. Suppose $A(f), A(g)$ and $A\left(f^{-1}\right)$ are Sonnenschein matrices with $A(f)$ a regular, one-to-one transformation. Then $c_{A(f)} \subset c_{A(g)}$ and $A(f) x$ and $A(g) x$ converge to the same limit if and only if $D_{0}$ $\supset f^{-1}(\bar{\Delta}(0,1))$ and $A(g) \cdot A\left(f^{-1}\right)$ is regular.

Proof. Sufficiency. Since $A(f)$ is a regular Sonnenschein matrix and $A\left(f^{-1}\right)$ is Sonnenschein, a result of Bajšanski [2] implies $f(\bar{\Delta}(0,1))$ $\subset \bar{\Delta}(0,1) \subset D_{f^{-1}}$. Hence by the corollary to Theorem $1 A\left(f^{-1}\right)$ $=\{A(f)\}^{-1}$ and $A(g) \cdot A\left(f^{-1}\right)=A\left(g \circ f^{-1}\right)=A(g) \cdot\{A(f)\}^{-1}$ and $A(g)\left(A\left(f^{-1}\right) y\right)=\left(A(g) \cdot A\left(f^{-1}\right)\right) y, \quad A(f)\left(A\left(f^{-1}\right) y\right)=\left(A(f) \cdot A\left(f^{-1}\right)\right) y=y$, for all $y \in m$. Thus Theorem 2 implies the result.

Necessity. By Theorem 2 it remains only to show that $D_{0}$ $\supset f^{-1}(\bar{\Delta}(0,1))$ is necessary. Suppose $c_{A(f)} \subset c_{A(0)}$ and $D_{0} D f^{-1}(\bar{\Delta}(0,1))$ $=\left\{z:|f(z)|<1, z \in D_{f}\right\}$. By Lemma 1 this implies $D A(g) D D A(f)$ and this contradicts the hypothesis $c_{A(f)} \subset c_{A(o)}$. 
Let $f(z)=\left\{\alpha^{\prime}+\left(1-\alpha^{\prime}-\beta^{\prime}\right) z\right\} /\left(1-\beta^{\prime} z\right)$ and

$$
g(z)=\{\alpha+(1-\alpha-\beta) z\} /(1-\beta z)
$$

with $|\beta|<1$ and $\left|\beta^{\prime}\right|<1$. Then $A(f)=K\left(\alpha^{\prime}, \beta^{\prime}\right)$ and $A(g)=K(\alpha, \beta)$. When no confusion can arise $K\left(\alpha^{\prime}, \beta^{\prime}\right) \cap m \subset K(\alpha, \beta)$ will replace the more cumbersome $c_{K\left(\alpha^{\prime}, \beta^{\prime}\right)} \cap m \subset c_{K(\alpha, \beta)}$.

Theorem 4. Suppose $|\beta|<1,\left|\beta^{\prime}\right|<1$ and

$$
\begin{aligned}
|| \alpha^{\prime} \beta^{\prime}-1+\alpha^{\prime}+\beta^{\prime}|-2| \alpha^{\prime} \beta^{\prime}|\cos \theta| \\
\quad \geqq 2\left|\beta^{\prime}\right|\left|\frac{\alpha^{\prime}+\beta^{\prime}-1-\alpha^{\prime} \beta^{\prime}}{\alpha^{\prime}+\beta^{\prime}-1+\alpha^{\prime} \beta^{\prime}}\right|
\end{aligned}
$$

where $\theta$ is the positive angle between $\alpha^{\prime}$ and $\mu=\left(\alpha^{\prime} \beta^{\prime}-1+\alpha^{\prime}+\beta^{\prime}\right) / 2 \beta^{\prime}$ and

$$
1-\left|\alpha^{\prime}\right|^{2}>\left(1-\bar{\alpha}^{\prime}\right)\left(1-\beta^{\prime}\right)>0 \text { or } \alpha^{\prime}=\beta^{\prime}=0
$$

then $K\left(\alpha^{\prime}, \beta^{\prime}\right) \cap m \subset K(\alpha, \beta)$ and the transformed limits are the same if and only if

$$
\left.|\beta||1-| \alpha^{\prime}\right|^{2}|<|\left|\beta^{\prime}\right|^{2}-\left|1-\beta^{\prime}-\alpha^{\prime}\right|^{2} \mid
$$

and

$$
\left|\left(1-\beta^{\prime}\right)-\alpha^{\prime}(1-\beta)\right|^{2}-\left|\alpha\left(1-\beta^{\prime}\right)-\alpha^{\prime}(1-\beta)\right|^{2}
$$

$$
>(1-\bar{\alpha})(1-\beta)\left(1-\bar{\beta}^{\prime}\right)\left(1-\alpha^{\prime}\right)>0
$$

or $\alpha=\alpha^{\prime}$ and $\beta=\beta^{\prime}$.

Proof. If $|\beta|<1,\left|\beta^{\prime}\right|<1$, then $A(f)=K\left(\alpha^{\prime}, \beta^{\prime}\right)$ and $A(g)=K(\alpha, \beta)$ are Sonnenschein matrices and moreover it follows that the $K\left(\alpha^{\prime}, \beta^{\prime}\right)$ transform is one-to-one on $c_{K\left(\alpha^{\prime}, \beta^{\prime}\right)} \cap m$. For $A\left(f^{-1}\right)$ to be Sonnenschein it is necessary that the range of $f$ include the unit disk, i.e.

$$
f\left(\left\{z:|z|<1 /\left|\beta^{\prime}\right|\right\}\right) \supset \bar{\Delta}(0,1) .
$$

$f$ transforms the disk $D\left(0,1 /\left|\beta^{\prime}\right|\right)$ conformally onto the half plane, $H$, whose boundary contains $f\left(-1 / \beta^{\prime}\right)=\mu$ and whose interior contains $f(0)=\alpha^{\prime}$. The line through $\mu$ and $\alpha^{\prime}$ is thus perpendicular to the boundary of $H$, because the line through $-1 / \beta^{\prime}$ and 0 is perpendicular to the circle $C\left(0,1 /\left|\beta^{\prime}\right|\right)$. A simple calculation shows $H \supset \bar{\Delta}(0,1)$ if and only if

$$
|\mu||| \mu|-| \alpha^{\prime}|\cos \theta| \geqq\left|\mu-\alpha^{\prime}\right|,
$$

where $\mu=\left\{\alpha^{\prime} \beta^{\prime}-1+\alpha^{\prime}+\beta^{\prime}\right\} / 2 \beta^{\prime}$, if and only if (i) holds. 


$$
\begin{aligned}
& f^{-1}(z)=\left(z-\alpha^{\prime}\right) /\left\{\beta^{\prime} z+\left(1-\alpha^{\prime}-\beta^{\prime}\right)\right\} \\
&=\frac{\left(\frac{-\alpha^{\prime}}{1-\alpha^{\prime}-\beta^{\prime}}\right)+\left(1-\left[\frac{-\alpha^{\prime}}{1-\alpha^{\prime}-\beta^{\prime}}\right]-\left[\frac{-\beta^{\prime}}{1-\alpha^{\prime}-\beta^{\prime}}\right]\right) z}{1-\left(\frac{-\beta^{\prime}}{1-\alpha^{\prime}-\beta^{\prime}}\right) z}
\end{aligned}
$$

thus by the corollary to Theorem 1 ,

$$
\{A(f)\}^{-1}=A\left(f^{-1}\right)=K\left(\frac{-\alpha^{\prime}}{1-\alpha^{\prime}-\beta^{\prime}}, \frac{-\beta^{\prime}}{1-\alpha^{\prime}-\beta^{\prime}}\right)=K^{-1}\left(\alpha^{\prime}, \beta^{\prime}\right)
$$

if $\left|\beta^{\prime}\right|<1$ and (i) holds.

By Theorem 3, if $D_{0} \supset f^{-1}(\bar{\Delta}(0,1))$ then $A(g) \cdot A\left(f^{-1}\right)=A\left(g \circ f^{-1}\right)$ $=K(\alpha, \beta) \cdot K^{-1}\left(\alpha^{\prime}, \beta^{\prime}\right)$ which implies

$K(\alpha, \beta) K^{-1}\left(\alpha^{\prime}, \beta^{\prime}\right)=K\left(\frac{\alpha\left(1-\beta^{\prime}\right)-\alpha^{\prime}(1-\beta)}{1-\alpha^{\prime}-\beta^{\prime}+\beta \alpha^{\prime}}, \frac{\beta-\beta^{\prime}}{1-\alpha^{\prime}-\beta^{\prime}+\beta \alpha^{\prime}}\right)$.

Sledd [6] proved $K\left(\alpha^{*}, \beta^{*}\right)$ is regular if and only if $\alpha^{*}=\beta^{*}=0$ or $1-\left|\alpha^{*}\right|^{2}>\left(1-\bar{\alpha}^{*}\right)\left(1-\beta^{*}\right)>0$. Thus $K\left(\alpha^{\prime}, \beta^{\prime}\right)$ is regular if and only if (ii) holds and $K(\alpha, \beta) \cdot K^{-1}\left(\alpha^{\prime}, \beta^{\prime}\right)$ is regular if and only if (iv) holds.

Finally, $D_{o} \supset f^{-1}(\bar{\Delta}(0,1))$ if and only if

(1) $\frac{\left.|1-| \alpha^{\prime}\right|^{2}-\left(1-\bar{\alpha}^{\prime}\right)\left(1-\beta^{\prime}\right)|+|\left(1-\bar{\alpha}^{\prime}\right)\left(1-\beta^{\prime}\right) \mid}{\left.|| \beta^{\prime}\right|^{2}-\left|1-\alpha^{\prime}-\beta^{\prime}\right|^{2} \mid}<\frac{1}{|\beta|}$,

since $f^{-1}(\bar{\Delta}(0,1))$ is a disk with center,

$$
C=\frac{1-\left|\alpha^{\prime}\right|^{2}-\left(1-\alpha^{\prime}\right)\left(1-\bar{\beta}^{\prime}\right)}{\left|\beta^{\prime}\right|^{2}-\left|1-\alpha^{\prime}-\beta^{\prime}\right|^{2}}
$$

and radius

$$
R=\frac{\left|1-\beta^{\prime}\right|\left|1-\alpha^{\prime}\right|}{\left.|| \beta^{\prime}\right|^{2}-\left|1-\alpha^{\prime}-\beta^{\prime}\right|^{2} \mid}
$$

and (1) is equivalent to $|C|+|R|<1 /|\beta|$. Thus the transformed disk is contained in $D_{o}=\{z:|z|<1 /|\beta|\}$ if and only if (1) holds. Thus if (ii) holds (iii) is equivalent to (1).

The following corollaries to Theorem 4 give necessary and sufficient conditions for inclusion of some well-known matrix transforms by other matrix methods. In particular they answer some questions posed by Schoonmaker [5]. 
Corollary 1. If $\frac{1}{2}<p \leqq 1$ and $0<r<1$, then $E(p) \subset T(r)$ if and only if $(1-p) /(2-p)<r<p /(2-p)$.

Proof. With the notation of Theorem $4, E(p)=K(1-p, 0), T(r)$ $=K(0, r)$. Thus conditions (i) and (ii) of that theorem are satisfied, because (i) is trivially true and (ii) is equivalent to $0<p \leqq 1$. Condition (iii) with hypothesis $\frac{1}{2}<p \leqq 1$ and $0<r<1$ becomes $|r||1-| 1-\left.\left.p\right|^{2}|<| p\right|^{2}$. This holds if and only if

$$
r<p /(2-p) \text {. }
$$

Condition (iv) with $\frac{1}{2}<p \leqq 1$ and $0<r<1$ becomes

$$
|1-(1-p)(1-r)|^{2}-|1(1-p)(1-r)|^{2}>(1-r) p>0
$$

which is equivalent to

$$
r>(1-p) /(2-p) \text {. }
$$

But inequalities (1) and (2) can hold simultaneously only if $\frac{1}{2}<p$ and thus the result follows.

CorollaRy 2. If $0 \leqq r \leqq \frac{1}{3}$ and $|q|<1$ then $T(r) \cap m \subset S(q)$ if and only if $0<q<1-2 r$.

Proof. $S(q)=K(1-q, q)$ and $T(r)=K(0, r)$. If $0 \leqq r \leqq \frac{1}{3}$ and $|q|<1$, condition (i) of Theorem 4 is satisfied, and (ii) is satisfied since (ii) is equivalent to regularity of $T(r)$ or $0 \leqq r<1$. Conditions (iii) and (iv) will be satisfied if and only if

$$
|q|<|| 1-\left.r\right|^{2}-|r|^{2} \mid
$$

and

$$
|1-r|^{2}-|(1-q)(1-r)|^{2}>\bar{q}(1-q)(1-r)>0 .
$$

It follows from $\bar{q}(1-q)(1-r)>0$ and $|q|<1$ that $q$ is real and $q>0$. But then, under the hypothesis of the corollary, (1) becomes $q<1-2 r$ and (2) becomes $q>(2 r-1) / r$. But this latter inequality is satisfied since $(2 r-1) / r<0<q$. Therefore (1) and (2) are equivalent to $0<q<1-2 r$.

CoRollaRY 3. If $0 \leqq r \leqq \frac{1}{3}$, then $T(r) \cap m \subset E(p)$ if and only if $0<p<1$.

Proof. $K(0, r)=T(r)$ and $K(1-p, 0)=E(p)$. If $0 \leqq r \leqq \frac{1}{3}$ then, a fortiori, $0 \leqq r<1$ which is equivalent to condition (ii) and implies condition (i) of Theorem 4. Conditions (iii) and (iv) of that theorem are equivalent to 


$$
0<\left.|| r\right|^{2}-|1-r|^{2} \mid
$$

and

$$
|1-r|^{2}-|(1-p)(1-r)|^{2}>p(1-r)>0,
$$

respectively. (1) is trivially satisfied and (2) implies $p$ is real, $p>0$. The first inequality in (2) thus reduces to $p<1$ and the result follows.

Corollaries 1, 2, and 3 strengthen and add new results to theorems of Schoonmaker [5]. In conclusion it should be noted that results for $E(p) \supset S(q)$ and $T(r) \supset S(q)$ could not be found using the methods of this paper because $f^{-1}(z)=\{z-(1-q)\} / q z, f(z)=(1-q) /(1-q z)$, $|q|<1$ is not analytic at the origin. This leads the author to suspect that $S^{-1}(q)$ does not exist but no results along these lines could be found.

\section{BIBLIOGRAPHY}

1. R. P. Agnew, Euler transformations, Amer. J. Math. 66 (1944), 313-338.

2. B. Bajšanski, Sur une classe générale de procédés de summations du type d'EulerBorel, Publ. Inst. Math. (Beograd) 10 (1956), 131-152.

3. G. Lausch, Relations among the Weierstrass methods of summability, Doctoral Dissertation, Cornell University, Ithaca, N. Y., 1949.

4. W. Meyer-König, Untersuchungen über einige verwandte Limitierungsverfahren, Math. Z. 52 (1949), 257-304.

5. N. Schoonmaker, Inclusion theorems among some methods of summability, Proc. Amer. Math. Soc. 7 (1956), 102-108.

6. W. T. Sledd, Regularity conditions for Karamata matrices, J. London Math. Soc. 38 (1963), 105-107.

7. J. Sonnenschein, Sur les series divergentes, Acad. Roy. Belg. Bull. Cl. Sci. 35 (1949), 594-601.

8. P. Vermes, Series to series transformations and analytic continuation by matrix methods, Amer. J. Math. 71 (1949), 541-562.

VILLANOVA UNIVERSITY 